Jpn. J. Phys. Fitness Sports Med. 2011, 60 : 99 112

\title{
CONSTRUCTION OF DELAYED MENARCHE EVALUATION IN KOREAN FEMALE ATHLETES
}

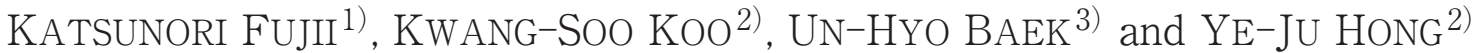

\begin{abstract}
In the present study we conducted a regression analysis of age at menarche against Maximum Peak Velocity (MPV) of height in non-athlete Korean girls which was derived with the wavelet interpolation method, and composed linear to quartic regression polynomials to obtain the best regression polynomial. We then applied the age at menarche and age at MPV of height of athletes to the best polynomial regression evaluation, and investigated the validity of a delayed menarche evaluation that we constructed. Moreover, the relation between delayed menarche and menstrual status was examined by investigating delayed menarche and menstrual pain in individuals for the first time. The subjects were 124 second year female students at a physical education high school in the suburbs of Pusan, South Korea. A questionnaire survey of these girls was conducted, from which their date of birth, age at menarche, and athletic activities in elementary, junior high, and high school were obtained. In addition, health check records were examined retrospectively, and longitudinal growth data for height were obtained from the 1st year of elementary school (7 years old) until the second year of high school (17 years old). Next, the same survey as above was also done for second year students at a general high school in the same area, as a control group. Three hundred forty-five non-athletes for whom all data were available were selected. As the results, the third order polynomial was found to be most suitable for the regression polynomial. When it was applied to individual female Korean athletes with respect to the regression evaluation, positive scores were obtained for many athletes and an overall delay in menarche was seen. Delayed menarche was not seen, however, in archery athletes. A strong delay in menarche was thus found in Korean athletes. And it was shown that $80 \%$ had moderate or greater menstrual pain and a close relation with menstrual abnormalities, the effectiveness of the delayed menarche evaluation was further validated.
\end{abstract}

(Jpn. J. Phys. Fitness Sports Med. 2011, 60 : 99 112)

Key words: Age at menarche, Delayed menarche, Age at Maximum Peak Velocity, Wavelet Interpolation Method

\section{I . Introduction}

Late menarche in female athletes has been reported by Malina ${ }^{1)}$ from menarche data on a large number of female Olympic athletes. Other reports by Malina et al. ${ }^{2 \sim 5)}$ have also shown that menarache was late in female athletes, suggesting delayed menarche. In Japan, Yamakawa et al. ${ }^{6)}$ have also suggested that menarche is delayed in female ath- letes. Malina and Bouchard ${ }^{7)}$ explained delayed menarche in female athletes with reference to the critical weight hypothesis of Frisch and Revelle ${ }^{8)}$ and Frisch et al. ${ }^{9)}$ That hypothesis states that the mechanism for delayed menarche is that the levels of gonadotropin circulating in blood change as a result of the higher proportion of fat-free mass with respect to fat mass that results from heavy training. Yamakawa et al. ${ }^{6)}$ conjectured
1) 愛知工業大学大学院経営情報科学研究科

干470-0392 愛知県豊田市八草町八千草1247

2) 昌原大學校體育學科

干641-773 慶向南道 昌原市士林洞 9番地

3) 慶南大學校體育學科

干631-701 晌南道 昌原市 馬山合浦區 月影洞449番地
Graduate School of Business Administration and Computer Science, Aichi Institute of Technology

1247, Yachigusa, Yakusacho, Toyota-city, Aichi

Department of Physical Education, Changwon National University Changwon City, Korea

Department of Exercise Science \& Sport Studies, Kyongnan University

Changwon City, Korea 
that there was an effect on hormone inhibition in the hypothalamus and pituitary gland from the stress of athletic training. However, Malina ${ }^{10)}$ also pointed out that female athletes have a tendency to mature late, and delayed menarche has not as yet been clearly demonstrated. To prove delayed menarche regardless of early or late maturation, it is necessary to establish criteria that objectively show delays in menarche. Fujii et al. ${ }^{11)}$ applied the age at maximum peak velocity of growth in height during puberty, which is not affected by training, in establishing these criteria. One problem was the method of specifying the age at maximum peak velocity of height during puberty. However, by specifying the age at maximum peak velocity (MPV) in height with the use of wavelet interpolation, Fujii ${ }^{11)}$ demonstrated delayed menarche from the interval between age at MPV of height and age at menarche. Then, by establishing delayed menarche judgments in individuals from regression analysis of age at menarche with respect to age at MPV of height, Fujii ${ }^{12)}$ developed a system to evaluate delayed menarche. After delayed menarche had been demonstrated, it was thought that its relation with menstrual irregularity and amenorrhea ${ }^{13 \sim 17)}$ could be clarified.

Fujii et al $^{18)}$ applied the delayed menarche evaluation system to general Japanese females, and examined menstrual status after menarche and exercise status around the age of menarche in individuals who were judged to have delayed menarche. As a result, a close relationship between exercise status and delayed menarche could be derived, but this relationship was not implicated in menstrual status. These findings may be considered significant in terms of the relationship between delayed menarche and menstruation status after menarche and exercise status around the age of menarche, based on the delayed menarche evaluation. Thus, by constructing a method to evaluate delayed menarche, a link can be shown between delayed menarche and menstrual status after menarche in individuals, and menstrual status and the possibility of pregnancy can be investigated. This poses important questions about female physiology and the warning of hard sports training around the age of menarche. This type of study should be conducted not only in Japan, but also in Korea. This is because, obviously, the problem of delayed menarche in female athletes also occurs in Korea. It is a problem that is already occurring with the popularity of sports in Asian countries in recent years. There are few studies such as this even in Japan, and in the future this kind of study should be undertaken in other countries including Korea.

This study therefore examined whether or not the same logic used to test delayed menarche in female athletes in Japan by Fujii ${ }^{11)}$ could be applied to female athletes in Korea. A structure was also created to clarify the relation between delayed menarche and menstrual irregularity or menstrual pain by analyzing the menstrual status of individuals judged to have delayed menarche. This requires construction of a system to evaluate delayed menarche in female athletes in Korea. Therefore, the interval between the age at menarche and age at MPV of height in general Korean females was analyzed in order to establish a system of delayed menarche evaluation, and a five-stage mean value evaluation in the interval was constructed. As indicated by Fujii and Demura ${ }^{19)}$, the difference between age at menarche and age at MPV of height becomes gradually smaller as the age at MPV of height increases. Thus, the evaluation in the early and late maturation zones may differ. Therefore, to improve the accuracy of the evaluation of delayed menarche proposed by Fujii and Demura ${ }^{20)}$, data on general Korean females were obtained and the validity of the polynomial from the first to the fourth order was examined with regard to the regression evaluation of the age at menarche for age at MPV of height. A delayed menarche evaluation chart was constructed by deriving a regression evaluation of the most appropriate order. This regression evaluation was applied to various sports to judge delayed menarche in athletes. Specifically, 
by investigating delayed menarche and menstrual status in individuals in this study, the relation between delayed menarche and menstrual status in female athletes was examined for the first time. The effectiveness of building a delayed menarche evaluation is therefore advocated, and the warning to Asian female athletes' abnormal menstruation including the South Korean female athletes is to be sounded.

\section{Methods}

\section{A. Subjects}

One hundred thirty female high school students in the Pusan region of South Korea, all of whom had participated in national sport competitions at the high school level (athletes group), were chosen as subjects. Consent was obtained for the inspection of health examination records, the use of measurement data on height and weight, and participation in a questionnaire survey. Longitudinal height and weight records of the subjects for the period from the first grade of elementary school to the second year of high school (1997, 1998-2008, 2009) were used. The age at menarche was confirmed according to the method of Malina et al. ${ }^{5)}$ and Mesaki et al. ${ }^{21)}$, which is used internationally. After consent from each subject had been obtained, we confirmed ages down to units of months in an interview (calculated to the first decimal place for each age). Height and weight were limited to those measured in May, and data in which the age at menarche could not be confirmed to the month were not used. Finally, 124 subjects for which data were complete were used for the analysis.

The sports engaged by the 124 female athletes were as follows (numbers of individuals are in parentheses): Judo (11), track and field (24), table tennis (10), badminton (4), hockey (3), swimming (8), taekwondo (15), gymnastic (12), archery (8), shooting (10), wrestling (3), weight lifting (7) and fencing (9). All athletes were prizewinners in prefectural level competitions, and had been selected for national competitions. All had trained about three to four hours a day, six days a week from the beginning of junior high to the end of senior high school (ages 12 to 17). According to the questionnaires and interview responses, their previous history of training had in most cases started from about the fifth grade of elementary school (age 10).

For the control group, four hundred senior high school female students in the Pusan region of South Korea were chosen as general subjects. Consent was obtained for the inspection of health examination records, the use of measurement data on height and weight, and participation in a questionnaire survey. Longitudinal height and weight records of the subjects for the period from the first grade of elementary school to the second year of high school (1997-2008) were used. The age at menarche was confirmed according to the method as same as female athletes. After consent from each subject had been obtained, ages down to units of months was confirmed in an interview (calculated to the first decimal place for each age). Height and weight were limited to those measured in May, and data in which the age at menarche could not be confirmed to the month were not used. Finally, 345 subjects for which data were complete were used for the analysis. The study was conducted after receiving approval from the research ethics committee (S-No1) at Chanwong National University.

\section{B. Survey of menstrual status}

In this study, menstrual status was taken to be indicated by menstrual pain, and menstrual status was understood from the level of pain during menstruation. A visual analogue scale (VAS) was adopted to objectively measure the severity of pain. The VAS followed the method proposed by Keele. $^{22)}$ A $10 \mathrm{~cm}$ line was drawn on a piece of white paper with $1 \mathrm{~cm}$ increments. With the left end as no pain and the right side as intense pain, the subjects drew a mark at the place on the line 
that corresponded to the pain they were feeling at the moment. Scores were given in the range of $0-10$, with higher scores indicating higher levels of menstrual pain.

\section{Analytical techniques}

To approximate the true growth curve from the supplied growth data with the wavelet interpolation method (WIM), the data are interpolated from the wavelet function of the data and a growth distance curve is drawn. This distance curve is differentiated to arrive at the growth velocity curve, and the growth distance value of the age at menarche and the puberty peak is examined. The WIM can sensitively read local phenomena, and has an extremely high level of approximation accuracy. The theoretical background and grounds for its efficacy have been described in previous reports ${ }^{23 \sim 25)}$. The procedures based on the WIM were applied to height values from 7 to 17 years of age in the Korean female athlete and control groups. First, WIM was applied to the age distance value of height for each individual. Then, the age distance curves for height in both groups were differentiated, and the age at MPV of height (age at peak during puberty) from the velocity curve was specified through this process. In addition, the interval between age at menarche and age at MPV of height was calculated for each person.

\section{Analytical procedures}

1) First, the MPV of height as the index of maturity rate is derived by the WIM in athletes and control groups.

2) Then, the interval is calculated between the age at menarche and the age at MPV of height, the mean evaluation in five stages is constructed by the mean and standard deviation, and the mean value evaluation is applied to female athletes.

3) A regression analysis of the age at menarche for the age at MPV of height is executed, the polynomial from the first to the fourth order is constructed, and the validity of the optimal order is examined.

4) The regression evaluation of the age at menarche for the age at MPV of height is constructed from the best-order polynomial and applied to female athletes.

5) A comparison is made between results of the mean evaluation and the regression evaluation applied to female athletes.

6) Delayed menarche was evaluated in each athlete, and the status of delayed menarche was investigated for each sport.

7) Menstrual status was investigated in athletes judged to have delayed menarche.

\section{E. Statistical methods}

An unpaired $t$-test was used for comparison of the athlete group and control group for the interval between age at MPV of height and age at menarche. A 5-step mean value evaluation method was applied to construct mean value evaluation in judging delayed menarche. For the validity of the order of the regression analysis polynomial, the method of judging from the residual sum of squares by Matsuura and Kim ${ }^{26)}$ and Largo et al. $^{27)}$ was applied simultaneously with the Akaike Information Criterion (AIC) and Bayesian Information Criterion (BIC). A $\chi^{2}$ test was used for the difference in distribution with the mean value evaluation method and regression evaluation method. In constructing the regression evaluation chart, a 5-step regression evaluation method was adopted with standard error from the regression plane as the standard criterion.

\section{$F$. Mean evaluation of interval between age at menarche and age at MPV of height \\ Normality test of interval between age at menarche and age at MPV of height}

The normal distribution function is shown as follows:

$$
f\left(x_{i}\right)=\frac{1}{\sqrt{2 \pi} \sigma} e^{-\frac{\left(x_{i}-\overline{\mathrm{x}}\right)}{2 \sigma^{2}}}
$$


The $\chi^{2}$ value is 34.4 , and it is inferred that there is no significant difference between the theoretical frequency and actual frequency of normal distribution in the $\chi^{2}$ test. Moreover, it was shown to be good fit to normal distribution.

Applying mean evaluation to interval between age at menarche and age at MPV of height

The difference in the interval between age at menarche and age at MPV of height is a reliable indicator of delayed menarche. The mean evaluation index was derived from a statistical analysis of the interval in non-athletes.

$\begin{array}{cccccc}\text { Early menses } & -2 & & & \text { interval } & <-1.18 \\ \text { Somewhat early } & -1 & -1.18 & \leqq & \text { interval } & <0.14 \\ \text { Normal } & 0 & 0.14 & \leqq & \text { interval } \leqq & 1.46 \\ \text { Somewhat delayed } & 1 & 1.46 & <\text { interval } \leqq 2.78 \\ \text { Delay } & 2 & 2.78 & <\text { interval }\end{array}$

If this evaluation method is applied to an athlete according to the given sport, a standard for delayed menarche can be derived. Fig 1, 2 shows the frequency distribution of evaluation score when the method was applied to female athletes. Therefore, the mean evaluation method clearly shows delayed menarche of Korean female athlete.

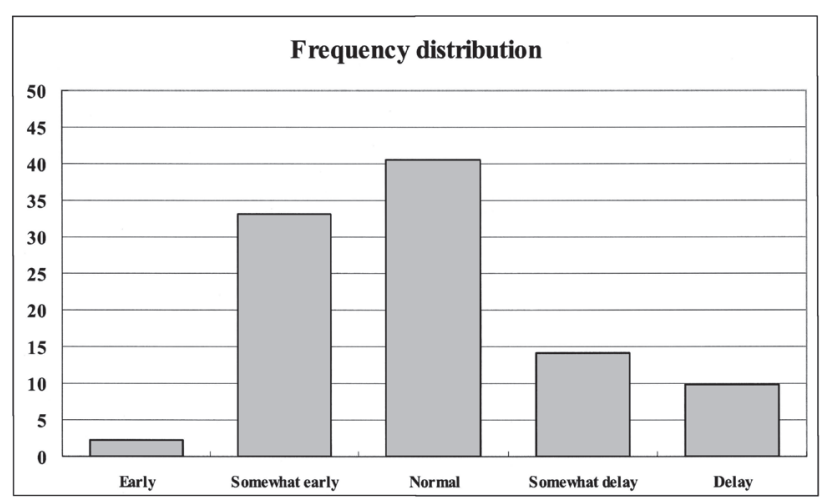

Fig 1 Frequency distribution derived from mean evaluation method in control group

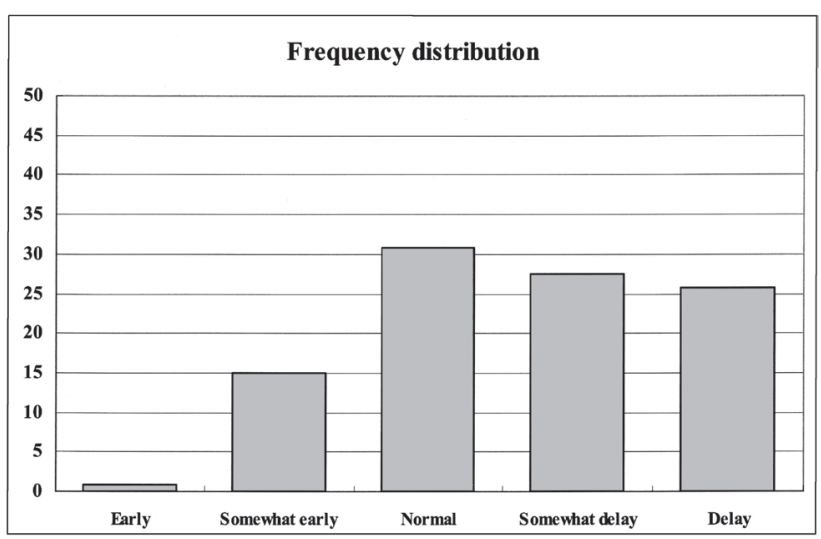

Fig 2 Frequency distribution derived from mean evaluation method in athletes group

\section{Results}

A. Comparison among Age at menarche, age at MPV of height and the interval between them in athlete and control groups

Age at menarche, age at MPV of height and the interval between them in athlete and control groups are shown in Table 1. In the athletes group, the average age at menarche was $13.6 \pm 1.51$ years, the average age at MPV of height was $11.7 \pm 1.18$ years and the interval between age at menarche and at MPV of height was $1.9 \pm 1.76$ years. When comparing with age at menarche $(12.4 \pm 1.12)$, age at MPV of height $(11.6 \pm 1.29)$ and the interval between age at menarche and age at MPV of height in the control group $(0.8 \pm 1.32)$, statistical analysis revealed significant differences $(\mathrm{p}<0.01)$ between the two groups in age at menarche and the interval between age at menarche and age at MPV of height. Therefore, physical maturity rates in athletes and control groups tended to be similar judging from the age at MPV of height, however, the menarche is delayed and delayed menarche was shown in athletes.

B. Validity of regression analysis for evaluating delayed menarche

Validity of delayed menarche evaluated by least squares approximation polynomial analysis 
Table 1 Statistics of age at menarche, age at MPV of height, interval between age at menarche and at MPV of height in Korean sport athletes and control groups

\begin{tabular}{lccccc}
\hline & & Age at menarche & $\begin{array}{c}\text { Age at MPV of } \\
\text { height }\end{array}$ & Interval & N \\
& & (years) & (years) & (years) & \\
\hline \multirow{2}{*}{ Sports athletes } & mean & $13.6^{* *}$ & 11.7 & $1.9 * *$ & 120 \\
& SD & 1.51 & 1.18 & 1.76 & \\
\hline \multirow{2}{*}{ Control } & mean & 12.4 & 11.6 & 0.8 & 345 \\
& SD & 1.12 & 1.29 & 1.32 & \\
\hline \hline
\end{tabular}

\section{Example 1. A linear least squares approximation}

The correlation coefficient was 0.4064 , and this was significant $(\mathrm{P}<0.01)$ in the non-athletes. Accordingly, we applied a regression analysis to the age at menarche and age at MPV of height. Thus, the primary polynomial was derived from the least squares approximation as follows:

$\mathrm{y}=0.3534 \mathrm{x}+8.284(\mathrm{SE}=1.03) \quad(\mathrm{y}=$ age at menarche; $\mathrm{x}=$ age at MPV of height)

The residual sum of the squares for this regression was computed to derive the validity of the primary polynomial (regression equations) as shown in Table 2 and Fig. 3.

\section{Example 2. Quadratic least-squares approximation}

The least-squares approximation by the quadratic polynomial was applied to the regression estimation of the age at menarche and MPV of height in the non-athlete group as shown in Example 1 .

$\mathrm{y}=0.0967 \mathrm{x}^{2}-1.855 \mathrm{x}+20.726(\mathrm{y}=$ age at menarche; $\mathrm{x}=$ age at MPV of height)

The residual sum of squares for this regression was computed to derive the validity of the quadratic polynomial (regression equations) as shown in Table 2 and Fig. 4.

\section{Example 3. Cubic least-squares approximation}

The least-squares approximation by the cubic polynomial was applied to the regression estimation of age at menarche and of MPV of height in the non-athlete group as shown in Example 1. $\mathrm{y}=-0.0045 \mathrm{x}^{3}+0.521 \mathrm{x}^{2}-3.61 \mathrm{x}+27.25(\mathrm{y}=$ age at menarche; $\mathrm{x}=$ age at MPV of height)

The residual sum of the squares for this regression was computed to derive the validity of the cubic polynomial (regression equations) as shown in Table 2 and Fig. 5.

\section{Example 4. quartic least-squares approximation}

The least-squares approximation by the quaritic polynomial was applied to the regression estimation of age at menarche and of MPV of height in the non-athlete group as shown in Example 1.

$\mathrm{y}=-0.0149 \mathrm{x}^{4}+0.6812 \mathrm{x}^{3}-11.469 \mathrm{x}^{2}+84.29 \mathrm{x}-$ 216.67 ( $\mathrm{y}=$ age at menarche;

$$
\mathrm{x}=\text { age at MPV of height) }
$$

The residual sum of the squares for this regression was computed to derive the validity of the quartic polynomial (regression equations) as shown in Table 2 and Fig. 6.

The validity of the cubic polynomial was the highest judging from the results of the residual sum of the squares and AIC in linear, quadratic, cubic and quartic least-square approximation polynomials. Moreover, the result did not change though judged from BIC. Therefore, the cubic polynomial regression can estimate age at menarche for age at MPV of height when evaluating delayed menarche.

Application of age at menarche for age at MPV of height to regression evaluation

The cubic polynomial regression graph of age at 
Table 2 Least square approximation polynomial and residual sum of square, AIC and BIC

\begin{tabular}{|c|c|c|c|c|}
\hline & Expression & $\begin{array}{c}\text { Residual } \\
\text { sum of } \\
\text { square }\end{array}$ & AIC & BIC \\
\hline Linear & $y=0.3535 x+8.2841$ & 362.6 & 1396.5 & 1394.1 \\
\hline Quadratic & $y=0.0967 x^{2}-1.8545 x+20.726$ & 340.7 & 1386.9 & 1386.2 \\
\hline Cubic & $y=-0.00450 x^{3}+0.5209 x^{2}-3.61 x+27.25$ & 342.0 & 1378.2 & 1384.4 \\
\hline Quartic & $y=-0.0149 x^{4}+0.6812 x^{3}-11.469 x^{2}+84.29 x-216.67$ & 626.8 & 1426.5 & 1448.2 \\
\hline
\end{tabular}

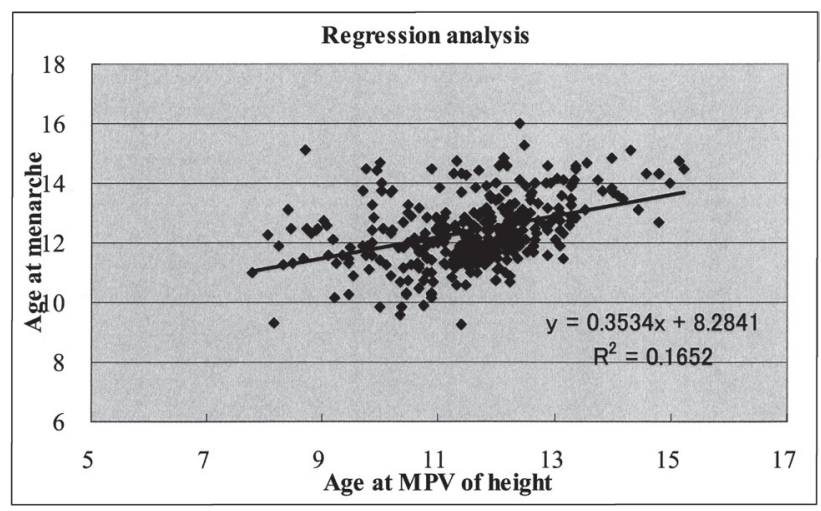

Fig 3 A least square approximation by linear polynomial

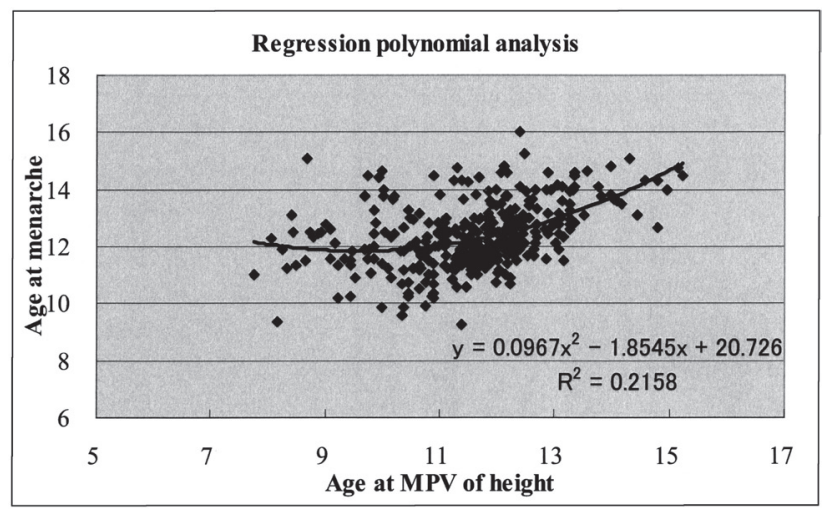

Fig 4 A least square approximation by quadratic polynomial

menarche for age at MPV of height was derived from control data as shown in Fig. 7. The cubic regression equation $\left(\mathrm{y}=-0.0045 \mathrm{x}^{3}+0.521 \mathrm{x}^{2}-3.61 \mathrm{x}\right.$ $+27.25)$ is described by applying the standard error $(=0.997)$ for this cubic polynomial fit. Since this cubic regression evaluation chart was applied to the athlete group (Fig 9), the standard for evalu-

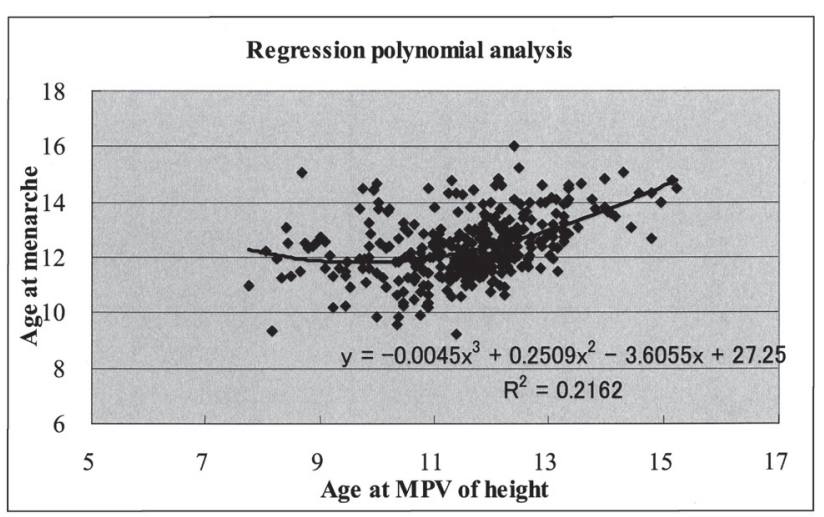

Fig 5 A least square approximation by cubic polynomial

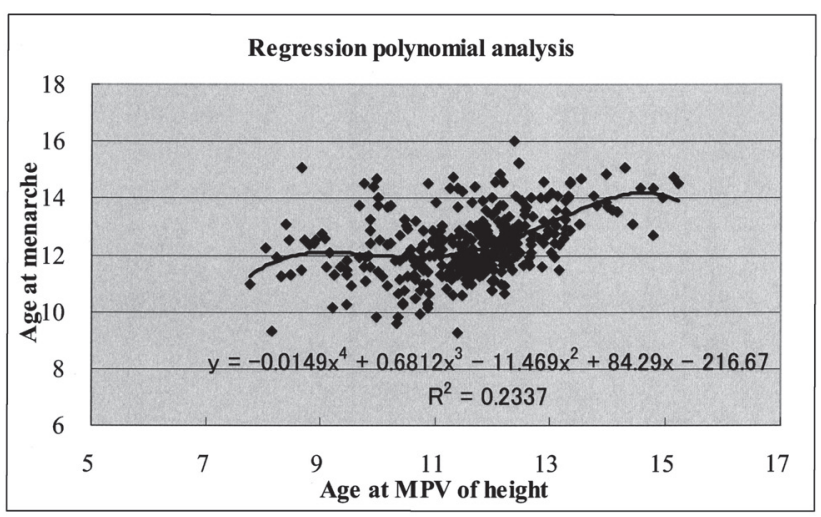

Fig 6 A least square approximation by quartic polynomial

ating delayed menarche may be as follows. When $+1.5 \sigma$ is exceeded, there is a delay. The interval between $+1.5 \sigma$ and $+0.5 \sigma$ indicates somewhat delay, and the regression line is the standard regression equation of age at menarche for age at MPV of height in the control group. The interval between $+0.5 \sigma$ and $-0.5 \sigma$ indicates the normal- 
ity, and the interval between $-0.5 \sigma$ and $-1.5 \sigma$ indicates somewhat early menarche. Below -1.5 $\sigma$ is judged to be early menarche. Fig. 9, 10 shows a case in which the cubic regression evaluation constructed on the basis of the control group was applied to the athletes group. Delayed menarche in the athletes was obviously confirmed when comparing with the score distribution of the control group.
Moreover, Table 3 showed the result of judging delayed menarche in each sports athletes. As shown in this Table 3, the delayed menarche was clearly shown in almost athletes, but archery athletes could not be judged with delayed menarche. As shown in Table 3, especially though the taekwondo and track and field were clearly judged with delayed menarche, the archery was not shown as delayed menarche.

Table 3 Result of delayed menarche judgment in each athletes

\begin{tabular}{|c|c|c|c|c|c|}
\hline & $\begin{array}{c}\text { Early } \\
\text { menses }\end{array}$ & $\begin{array}{c}\text { Somewhat } \\
\text { early }\end{array}$ & Normal & $\begin{array}{c}\text { Somewhat } \\
\text { delay }\end{array}$ & Delay \\
\hline Track and field & & 2 & 4 & 8 & 10 \\
\hline Hockey & & & 2 & & 1 \\
\hline Fencing & & 2 & & 3 & 4 \\
\hline Badminton & & 1 & 1 & 1 & 1 \\
\hline Taekwondo & & & 3 & 3 & 8 \\
\hline Gymnastic & 1 & & & $\mathbf{1}$ & 7 \\
\hline Judo & 1 & & 2 & 3 & 5 \\
\hline Weight lifting & & & 3 & 1 & 3 \\
\hline Archery & & 3 & 3 & 2 & \\
\hline Table tennis & & 1 & 2 & 4 & 3 \\
\hline Shooting & & 1 & & 7 & 2 \\
\hline Wrestling & & & 1 & & 2 \\
\hline Swimming & & 1 & 2 & 4 & 1 \\
\hline
\end{tabular}

Amenorrhea: Three gymnasts, one Taekwondo (Not include the above table)

Table 4 Comparison between mean evaluation and regression evaluation applied to control and athletes groups

\begin{tabular}{c|c|c|c|c|c|c|}
\multicolumn{2}{c|}{} & $\begin{array}{c}\text { Early } \\
\text { menses }\end{array}$ & $\begin{array}{c}\text { Somewhat } \\
\text { early }\end{array}$ & Normal & $\begin{array}{c}\text { Somewhat } \\
\text { delay }\end{array}$ & Delay \\
\hline \multirow{3}{*}{ Control } & $\begin{array}{c}\text { Mean } \\
\text { evaluation }\end{array}$ & 8 & 114 & 140 & 49 & 34 \\
\cline { 2 - 7 } & $\begin{array}{c}\text { Regression } \\
\text { evaluation }\end{array}$ & 18 & 78 & 149 & 73 & 27 \\
\hline \hline \multirow{3}{*}{ Athletes } & $\begin{array}{c}\text { Mean } \\
\text { evaluation }\end{array}$ & 1 & 18 & 37 & 33 & 31 \\
\cline { 2 - 7 } & $\begin{array}{c}\text { Regression } \\
\text { evaluation }\end{array}$ & 2 & 11 & 23 & 37 & 47 \\
\hline
\end{tabular}

The $\chi^{2}$ test between mean and regression evaluation showed significant difference $(\mathrm{P}<0.05)$ in control and athletes groups. 


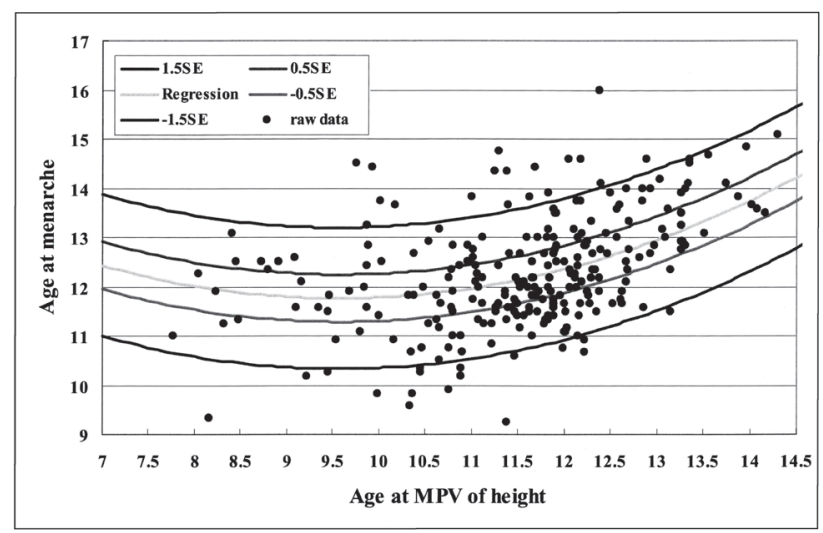

Fig 7 Delayed menarche evaluation chart in control group

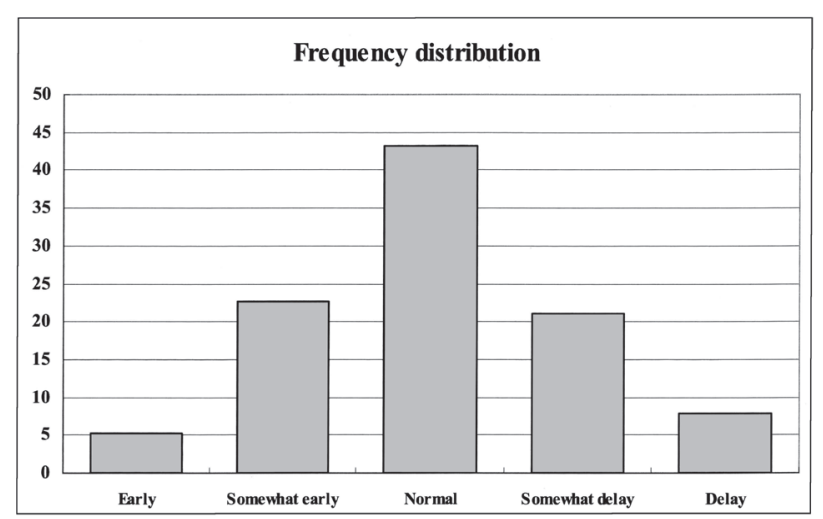

Fig 8 Frequency distribution of delayed menarche judgment by regression evaluation in control group

\section{Comparison between mean value evaluation and regression evaluation}

As shown in Table 4 and Fig. 1, 8, and Fig 2, 10 , it is the result of a judgment derived from application to all athletes for the mean value and regression evaluations. The $\chi^{2}$ test regarding the number determined by both evaluation methods showed significant difference $(\mathrm{P}<0.05)$. In other words, the mean evaluation level tended to be slightly higher in somewhat early menarche and to be lower in somewhat delay. Therefore, the cubic regression evaluation may be appropriate when strictly determining individual differences.

\section{Relation between delayed menarche and menstrual status}

In this study menstrual status was understood from menstrual pain. Menstrual pain is also called

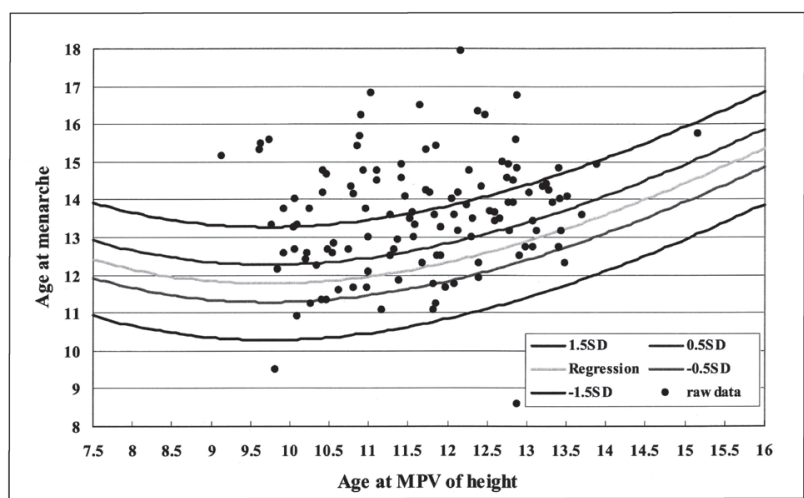

Fig 9 Delayed menarche evaluation chart in sport athletes group

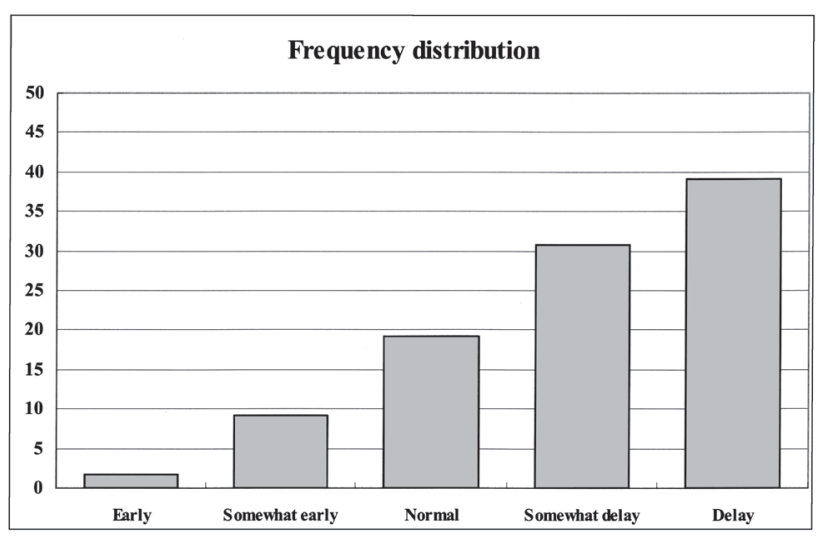

Fig 10 Frequency distribution of delayed menarche evaluation in athletes group

dysmenorrhea, with menstruation accompanied by lower abdominal pain and low back pain. This pain was measured using a VAS. Figure 11 shows the frequency distribution of the severity of menstrual pain in athletes. This distribution does not include 4 amenorrheic athletes. Judging from this distribution diagram, nearly $80 \%$ of athletes have menstrual pain of moderate or greater intensity. All athletes were classified based on judgments of delayed, regular, and early menarche, and distributions showing the intensity of menstrual pain in these groups are shown in Figs. 12-14 There were 84 girls with delayed or somewhat delayed menarche, 23 with regular menarche, and 13 with early or somewhat early menarche. Because of the larger number of girls in the delayed menarche group, a generalized comparison of the distribution in the 3 groups is difficult. However, in the delayed 
menarche group the intensity and frequency of menstrual pain closely resembled that in all athletes, indicating that the intensity and frequency of menstrual pain in the delayed menarche group directly reflects the intensity of menstrual pain in athletes. It is suggested, therefore, that delayed menarche in girls is closely related to menstrual abnormalities.

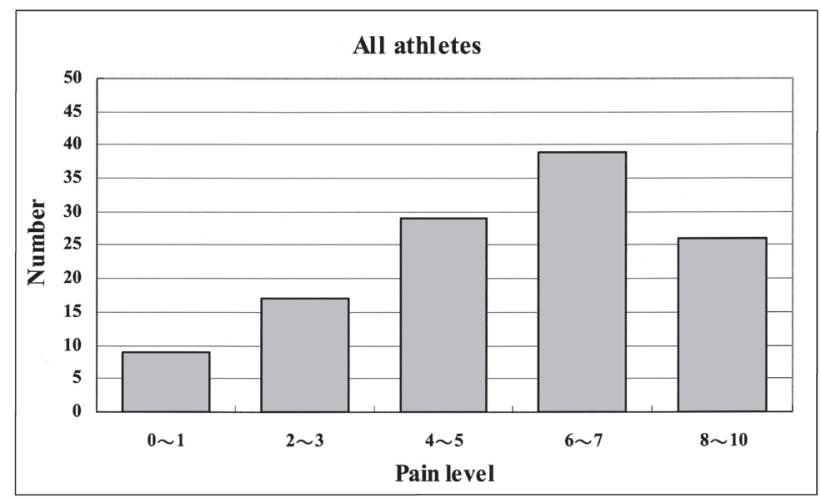

Fig 11 Frequency distribution of pain level of menstruation in all athletes

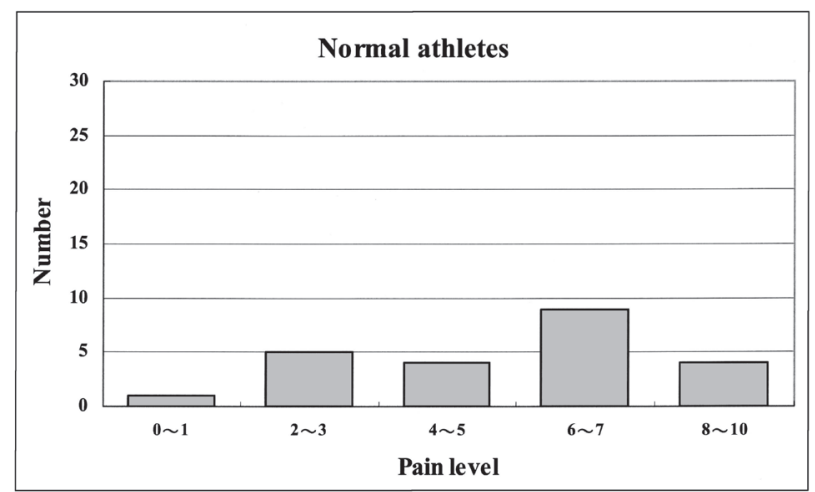

Fig 12 Frequency distribution of pain level of menstruation in normal athletes

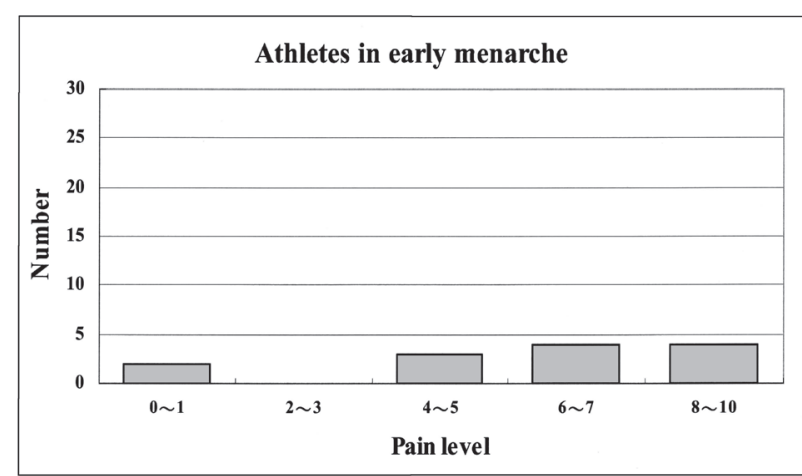

Fig 13 Frequency distribution of pain level of menstruation in early menarche athletes

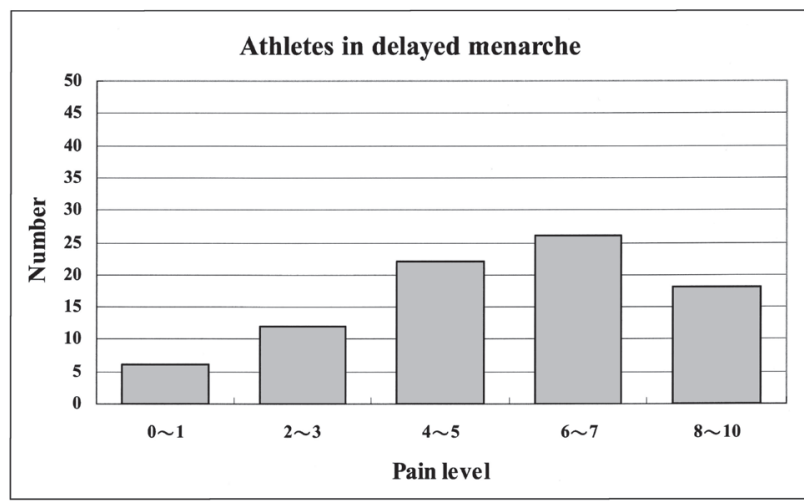

Fig 14 Frequency distribution of pain level of menstruation in delayed menarche athletes

\section{N. Discussion}

Delayed menarche in Korean female athletes can be verified by examining the interval between age at menarche and age at MPV of height using the WIM proposed by Fujii ${ }^{25)}$. Of course, regular training just before and after the age at menarche could not be assumed as a factor. However, other studies ${ }^{1,21,28 \sim 32)}$ have identified regular training as an important factor in delayed menarche. Accordingly, a procedure was needed to demonstrate delayed menarche among female non-athletes as well as female athletes in Japan (Fujii ${ }^{11)}$ ). In the present study, we attempted to construct a delayed menarche evaluation system to establish criteria with which to judge delayed menarche in Korean female athletes.

A mean evaluation method was applied from the mean intervals between the age at menarche and the age at MPV of height as a criterion for delayed menarche, and the delayed menarche in individual female athletes was obtained from this evaluation method. As understood from this result, positive scores in the evaluation by this method indicate delayed menarche among female athletes. Although delayed menarche can be determined to some extent by this mean method, important problems arise. That is why the interval between age at menarche and age at MPV of height changes according to the maturity rate as indicated by 
Fujii and Demura ${ }^{19)}$. In other words, the interval increases with early maturity and decreases with later maturity. Therefore, dealing with this situation is virtually impossible by the mean evaluation method. The validity of the cubic regression evaluation is clearly shown from the comparison between mean value and regression evaluation in the result of this study.

Therefore, a regression analysis was applied to age at menarche against age at MPV of height as an evaluation that considers level of maturity. When applying the regression analysis, age at MPV of height as the independent variable was also meaningful as an indicator of level of maturity. It was therefore effective in enabling estimation of age at menarche with direct consideration of level of maturity. However, when trying to estimate age at menarche from only age at MPV of height, which is a variate independent variable, fundamentally a least squares method is applied to estimate the curvilinear regression. In such cases application of a least squares approximation polynomial with a higher order independent variable to increase the precision of the estimate is considered to be valid. In this study, least squares approximation polynomials up to the fourth order were constructed, and their accuracy was investigated. Third order regression polynomials were found to be valid. From the above, a regression evaluation with a third order least squares approximation polynomial was constructed for age at menarche against age at MPV of height, and applied to athletes.

The educational system in Korea, unlike Japan's, has a separate special physical education course starting in junior high school, and the female athletes used in this study were students of a high school specializing in physical education. Moreover, they were national level athletes. The general female high school students were students of a regular school who did not belong to a sports club at school. Therefore, it was not necessary to consider the effects of exercise before menarche. With this background, the ability to establish a control group of 345 general high school girls was very meaningful. As a result of constructing a delayed menarche evaluation from this large control group, a third order regression polynomial was constructed with Korean girls. In contrast, a first order regression polynomial evaluation had been constructed for Japanese girls. This difference in order of the regression polynomial between Japanese and Korean girls is predicted to be related to the regression chart of age at menarche against age at MPV of height. Fundamentally, high order curvilinear regression is applied when attempting to raise the accuracy of estimates above the linear regression in regression analysis. Of course, if the accuracy of the fit becomes higher as a result raising the order, it is necessary to apply higher orders. However, if the accuracy of the fit does not increase much even when the order is raised, a low order linear regression evaluation is appropriate. A third order polynomial was applied to evaluate delayed menarche in Korean girls because it was thought that the accuracy of curvilinear regression would naturally be high. That was because the number of data was much higher than for Japanese girls, and the space of the 2-dimensional coordinate axes that make up the regression polynomial became larger from the relation of the later age at MPV of height and age at menarche than in Japanese. Additionally, while the Korean girls in this study share the same East Asian ancestry as Japanese girls, the interval between age at MPV of height and age at menarche is smaller than that reported by Fujii ${ }^{12)}$ for Japanese girls. This means that although the age at menarche is later than in Japanese girls, after the appearance of MPV of height menarche comes sooner than in Japanese girls. It may be inferred that this phenomenon affects the regression composition of age at MPV of height and age at menarche of Korean girls.

Therefore, in this study we applied the delayed menarche evaluation system using a third order regression polynomial to Korean athletes, and de- 
termined whether they had delayed menarche. Many athletes had delayed menarche, although no clear delayed menarche was seen in the archery athletes. Rather, delayed menarche could not be judged. This is thought to indicate that the effect on delayed menarche differs with type of sport and the training for it. Since archery is a special sport in which the athlete tries to hit a target, posture which stood for a long time with the unbalanced drawing leased condition of right and left continues. For that, though the training of leg strength and muscular power of upper half of body is necessary, as indicated by Mabuchi et al ${ }^{33)}$, because a lot of archery players do not do strength training so much, there are a lot of players who appeal for lumbago. Therefore, it differs from sports with hard training that uses muscles of the entire body, there is a suspicion that it may not affect delayed menarche. Citing the delayed menarche hypothesis of Malina and Bouchard, ${ }^{7)}$ it may be that the proportion of fat-free mass against fat mass does not increase with archery, and changes in the blood circulating levels of gonadotropin are not seen. However, considering the effects of the hormone inhibition in the hypothalamus and pituitary gland from stress caused by hard training that was described by Yamakawa et $\mathrm{al}^{6)}$ and Mesaki et al, ${ }^{21 \text { ) }}$ it is difficult to conclude whether the delay in menarche with sports other than archery is from the direct effects of decreased fat or from the effects of stress.

In this study a clear delay in menarche was also seen in various athletes from the delayed menarche evaluation chart, and the subsequent menstrual status of individuals judged to have delayed menarche was examined. This study was the first to attempt this kind of analysis. Menstrual status was judged by the intensity of menstrual pain in this study. The intensity and frequency of menstrual pain in all athletes and the intensity and frequency of menstrual pain in girls judged to have delayed menarche were very similar. Normally, menstrual pain is thought to be caused by uter- ine contraction from excessive secretion of prostaglandin, and Yanagida et al. ${ }^{34)}$ suggested that menstrual pain is affected by hard sports training. Kikuchi ${ }^{35)}$ thought that abnormal menstrual cycles in female athletes are caused inhibited secretion of gonadotropin-releasing hormone from the hypothalamus, and suppressed secretion of luteinizing hormone from the pituitary gland. The series of mechanisms in abnormal menstrual cycles are equally applicable as the mechanisms for delayed menarche. In this study we did not investigate menstrual cycles, but nearly all girls judged to have delayed menarche showed moderate or greater menstrual pain, suggesting the possibility of dysmenorrhea or abnormal cycles. Amenorrhea was already observed in 4 of the 124 female athletes in this study. Age at MPV of height was examined in 3 gymnasts and 1 Taekwondo athlete, and found to be 9.2 and 9.7 years old in two gymnasts, 14.1 years old in 1 gymnast, and 13.8 years old in the Taekwondo athlete. Thus, when menarche that should have occurred judging from physical maturity has not been observed, it can be judged to be primary amenorrhea.

Thus, there is a high possibility that hard sports training causes abnormalities in the physiology (menstruation) of female athletes. Of course, menstrual abnormalities will not occur in all sports athletes, and so there is a need to identify the types of conditions under which abnormalities may occur and the types of people in which they are more common. However, there have been no reports on this. Judging from the findings of the present study, there seems to be a high relation between delayed menarche and subsequent menstrual abnormalities. The occurrence of delayed menarche may therefore be taken as a parameter for menstrual abnormalities. Thus, there is a need for attention to later sports involvement in girls with delayed menarche. Kikuchi ${ }^{35)}$ stated that menstrual cycle abnormalities do not necessarily return to normal when training is stopped, and that the longer the period of sports training the more 
difficult is the return to normal. Even if that is the case, a judgment of delayed menarche may be a warning for continuing training in female athletes. The delayed menarche evaluation in Korean female athletes in this study further advances the delayed menarche evaluation established by Fuji ${ }^{12)}$ in Japanese girls, and leads to a relation with menstrual status following delayed menarche. The present findings will be useful for resolving problems related to menstrual abnormalities in female athletes.

\section{Conclusion}

In the present study, a third order polynomial regression analysis of age at menarche against age at MPV of height was valid in constructing a delayed menarche evaluation system for Korean athletes. When the delayed menarche in Korean female athletes is determined as an average value, a clear and significant difference with general girls was seen. The results of an investigation of the validity of mean value evaluations and regression evaluation showed the validity of regression evaluation, and the validity of regression evaluation was seen in the judgments of delayed menarche in individuals. Application of the delayed menarche evaluation to athletes clearly showed that many athletes had delayed menarche, although delayed menarche in archery athletes could not be judged. As a result of investigating the later menstrual status in girls judged to have delayed menarche, it was found that $80 \%$ had moderate or greater menstrual pain and a close relation with menstrual abnormalities, suggesting that delayed menarche may be a barometer for menstrual abnormalities. The demonstration of delayed menarche in Korean female athletes from the above suggests the validity of the delayed menarche evaluation.

(Accepted September. 13, 2010)

\section{References}

1) Malina R M. Menarche in athletes : a synthesis and hypothesis. Ann Hum Biol, 10, 1-24, 1983.

2) Malina R M, Harper A B, Avent H H, and Campbell D E. Age at menarche in athletes and non-athletes. Med Sci Sport, 5, 11-13, 1973.

3) Malina R M, and Moss K A H. Age at menarche and family characteristics of high school athletes and non athletes. In Psychological Perspectives in Youth Sports, edited by F.L. Smoll and R.E. Smith (Washington, D.C. Hemisphere Publishing Corporation), 1978.

4) Malina R M, Spirduso W W, Tate C, and Baylor A M. Age at menarche and selected menstrual characteristics in athletes at different competitive levels and in different sports. Med Sci Sport, 10, 218-222, 1978.

5) Malina R M, Bouchard C, Shoup R F, Demirjian A, and Lariviere G. Age at menarche, family size, and birth order in athletes at Montreal Olympic Games,1976. Med Sci Sport, 11, 354-358 1979.

6) Yamakawa J, Shibuya S and Yokozeki T. Status of menstruation and menarcheal age in female athletes. Showa 58 th Japan Sports Association Sport Medical Science Research Report, " Study on female sports aptitude - III - “ , 84-99, 1983.

7) Malina R M. and Bouchard C. Growth, Maturation, and Physical Activity. Champaign, IL Human Kinetics. 1991.

8) Frish R E, and Revelle R. Height and weight at menarche and a hypothesis of critical body weight and adolescent events. Science, 169, 397-399, 1970.

9) Frisch R E, Revelle R, and Cook S. Height, weight and age at menarche and the "Critical Weight" hypothesis. Science, 174, 1148-1149, 1971.

10) Malina R M. Physical activity and training : effects on stature and the adolescent growth spurt. Medicine and Science in Sports and Exercise, 26, 759-766, 1994.

11) Fujii K. Verification regarding delayed menarche in Japanese female athletes - Analysis by Wavelet Interpolation Method -. Japan J. Phys. Educ. Hlth. Sport Sci., 48 , 523-539, 2003.

12) Fujii K. Construction of Delayed Menarche Evaluation System in Japanese Female Athletes, J. Educ. Health Sci, 53, 273-285, 2008.

13) Frisch R E. Fatness of girls from menarche to age 18 years, with a monogram. Hum Biol, 48, 353-359, 1976.

14) Frisch R E, Wyshak G, and Vincent L. Delayed menarche and amenorrhea in ballet dancers, New England Medical Journal, 303, 17-19, 1980.

15) Frisch R E, Gotz - Welbergen AV, Mcarthur J W, Albright T, Witscht J, Bullen B, Birnholz J, Reed R $B$, and Hermann H. Delayed menarche and amenorrhea of college athletes in relation to age of onset of 
training. J A M A, 246, 1559-1563, 1981.

16) Sanborn C F, Martin B J, and Wagner W W. Is athletic amenorrha specific to runners? Am J Obstet Gynecol, 143, 859-861, 1982.

17) Speroff L, and Redwine D B. Exercise and menstrual function. Physician Sportsmed, 8, 42-52, 1980.

18) Fujii K. Confirmation Regarding Delayed and Early Menarche in Female during Adolescence based on Delayed Menarche Evaluation System : Relation to Custom Condition of Sports Activeties, J. Educ. Health Sci, 55, 241-250, 2010.

19) Fujii K, and Demura S. Confirmation of Delayed Menarche Based on Regression Evaluation of Age at Menarche for Age at MPV of Height in Female Ball Game Players, Environ. Health Prev. Med, 10, 48-54, 2005.

20) Fujii K, and Demura S. An Approach to Verifying Delayed Menarche in Japanese Female Athletes Analysis by wavelet interpolation method-, The Journal of Sports Medicine and Physical Fitness, 45, 580-593, 2005.

21) Mesaki N, Sasaki J, Shoji M, Iwasaki H, and Eda M. Delayed menarche followed by onset of athletic sports training. Acta obast Gynaec Jpn. 36, 49-56, 1984.

22) Keele K D. THE PAIN CHART, The Lancet, 252 (6514) 6-8, 1948.

23) Fujii K, and Yamamoto $Y$. The analysis of the growth velocity curve in height based upon the maturity rate. Jpn J Phys Fitness and Sports, 44 : 431438, 1995.

24) Fujii K, and Matsuura Y. Analysis of the growth velocity curve for height by the Wavelet Interpolation Method in children classified by maturity rate. Am J Hum Biol, 11, 13-30, 1999.

25) Fujii K. A scientific approach to growth and development - Physical information science for growth and development \& health - . Sankeisha: 2006, pp.112-173.

26) Matsuura $Y$ and Kim M. Analysis of physical growth by fitting the polynomial to the longitudinal growth distance data of individual ; age 6 to 17, Research Monograph issued by Growth and Development Research Institute of Health and Sport Sciences University of Tsukuba Japan, pp. 1-153, 1991.

27) Largo R H, Gasser T, Prader A, Statzle W, \& Huber $\mathrm{P}$ J. Analysis of the adolescent growth spurt using smoothing spline functions. Ann Hum Biol, 5, 421434, 1978.

28) Baker E R, Mathur R S, Kirk R F, and Williamson $\mathrm{H}$ O. Female runners and secondary amenorrhea: Correlation with age, parity, mileage, and plasma hormonal and sex-hormone-binding globulin concentrations. Fertil Steril, 36, 183-187, 1981.

29) Baker E R, Mathur R S, Landgrebe S C, Mooby L O, and Williamson $\mathrm{H}$ O. Plasma gonadotropins, prolactin and steroid hormone concentrations in female runners immediately after a long-distance run. Fertil Steril, 38, 38-41, 1982.

30) Colt E. W. D, Warlaw S. L and Frantz A. G. The effect of running on plasma $\beta$-endorphin. Life Science, 28, 1637-1640, 1981.

31) Cohen J L, Kim C S, May P B Jr. and Ertel N H. Exercise, body weight, and amenorrha in professional ballet dancers. Physician Sportsmed, 10, 92-101, 1982.

32) Warren M D. The effect of exercise on pubertal progression and reproductive function in girls. J Clin Endocrinol Metab, 51, 1150-1157, 1980.

33) Mabuchi H, Tobaru T, Fujino $M$ and Nagao M. An investigation of physical conditioning and injures in the Japanese national archery team, Kawasaki Medical Welfare Journal, 19, 461-464, 2010.

34) Yanagida $Y$, Yamada $K$ and Koikawa N. Effect of natto intake on menstruation-related syndrome amang sports science and humanities students, Juntendo Health and Sport Science Study, 12, 29-39, 2008.

35) Kikuchi J. Influence which sports training causes to menstruation (cycle), Journal of Health, Physical Education and Recreation, 50, 379-387, 2000. 